\title{
Effect of Orientation on Plastic Deformation Behavior of Yttria Stabilized Zirconia Single Crystal
}

\author{
Deock-Soo Cheong and Jeon-Kook Lee*† \\ Department of Materials Science and Engineering, Dankook University, Chunan-city, Chungnam 330-714, Korea \\ *Materials Science and Technology Research Division, Korea Institute of Science and Technology, Seoul 136-791, Korea
}

(Received November 3, 2009 : Received in revised form November 20, 2009 : Accepted December 1, 2009)

\begin{abstract}
Yttria stabilized zirconia single crystals show plastic deformation at high temperatures by activating dislocations. The plastic deformation is highly dependent on crystallographic orientation. When the samples were deformed at different orientations, stress-strain curves changed by operating different slip systems. The strength of samples was also highly dependent on crystallographic orientation, i.e., samples without yield drop showed higher strength than that of samples exhibiting yield drop. The slip systems in the sample deformed along $<112>,<111>$ and $<001>$ agreed with the theoretical values of the plastic deformation, following Schmid's Law. Dislocations play a major role in the plastic deformation of this crystal. At the early stages of plastic deformation, all samples exhibited dislocation dipoles and, in the later stages, dislocation interactions occurred by forming nodes, tangles and networks. In this study, three different orientations, [11-2], [111] and [001] were employed to explain the plastic deformation behavior. A microstructural analysis was performed to elucidate the mechanism of the plastic behavior of this crystal.
\end{abstract}

Key words yttria stabilized zirconia single crystals, plastic deformation, activating dislocations, crystallographic orientation.

\section{Introduction}

Yttria fully stabilized Zirconia (Y-CSZ) single crystal were deformed by dislocation movement during high temperature deformation which showed yield drop by activation secondary slip system. ${ }^{1-2)}$ And yield drop was occurred after the yield point by activating the secondary slip system when sample was deformed along $<112>$. The dislocation structures in this crystal were studied comprehensively by Cheong at al. ${ }^{3)}$ The yield drop can be occurred when dislocation movement in the sample were faster than cross head speed. ${ }^{4)}$ In the early stage of deformation, dislocation dipoles were formed by cross slip ${ }^{4)}$ and edge trapping mechanism. ${ }^{5)}$ Plastic deformation behavior, yield drop and dislocation structures were strongly dependent on temperature and strain-rate. ${ }^{6-7)}$ Deformation behavior is strongly dependent on crystallographic orientation of this crystal. When the sample was deformed along $<111>$, three $\{001\}<110>$ slip systems were activated, which have the same Schmid factor of 0.45 . In the early stage of deformation, it showed yield drop similar to the

Corresponding author

E-Mail : jkleemc@kist.re.kr (J. -K. Lee) sample deformed along $<112>$. However when the samples were deformed along [001], no yield drop was observed in stress-stain curve. Eight $\{111\}<110>$ slip system were activated, which has the same Schmid factor of 0.45 .

In this study, the effect of orientation on plastic behavior of Y-CSZ single crystal was investigated by compression tests and microstructural analysis of the sample deformed at three different orientations.

\section{Experimental procedures}

\subsection{Preparation of compression sample}

Y-CSZ single crystals were received from Ceres Company (Waltham, MA, USA) which were doped with $9.4 \mathrm{~mol} \%$ $\mathrm{Y}_{2} \mathrm{O}_{3}$ grown by skull melting process. The crystals were rod shape, about $40 \mathrm{~mm}$ diameter and $100 \mathrm{~mm}$ in length. The orientations of the sample were determined by Laue $\mathrm{X}$-ray back reflection. Three compress axes were $<112>$, [111] and [001] which to activated different slip systems, such as the primary slip system, $\{001\}<110>$, three $\{001]$ $<110>$ and eight $\{111\}<110>$ slip systems, respectively. The orientation of compression axes was determined by Laue X-ray back reflection technique and the size of sample was $3 \times 3 \times 8 \mathrm{~mm}^{3}$. 


\subsection{Deformation at high temperature}

Plastic deformation experiments were carried out in a screw-driven Instron machine equipped with compression apparatus, consisting of deformation rods surrounded by high temperature furnace. The cross head speed of the compression tests were $2.08 \times 10^{-6} \mathrm{sec}^{-1}$. The deformation tests were performed at $1400^{\circ} \mathrm{C}$ in the air

\subsection{TEM foil preparation}

Transmission Electron Microscopy (TEM) samples were prepared from the sample deformed. All the foils were cut parallel to the primary slip planes, $\{001\}$ for the sample deformed along $<112>$ and $<111>$, and for the sample deformed along [001], foils were parallel to the (111) planes in order to study the dislocation structures. Then the samples were polished and cut into $3 \mathrm{~mm}$ disk, dimple polished and finally ion-bean thinning following the conventional TEM sample preparation procedure.

\subsection{TEM observation}

The analysis of dislocation loops in this study was performed in Philips 400T, operating at $125 \mathrm{kV}$. Conventional diffraction contrast experiments using dynamical two-beam conditions ${ }^{8)}$ were carried out to determine the Burgers vector and study the dislocation structures and interaction. In theses two beam conditions, each of the Burgers vector can be determined from $\mathrm{g} \cdot \mathrm{b}$ analysis, with at least two $\mathrm{g} \cdot \mathrm{b}$ conditions.

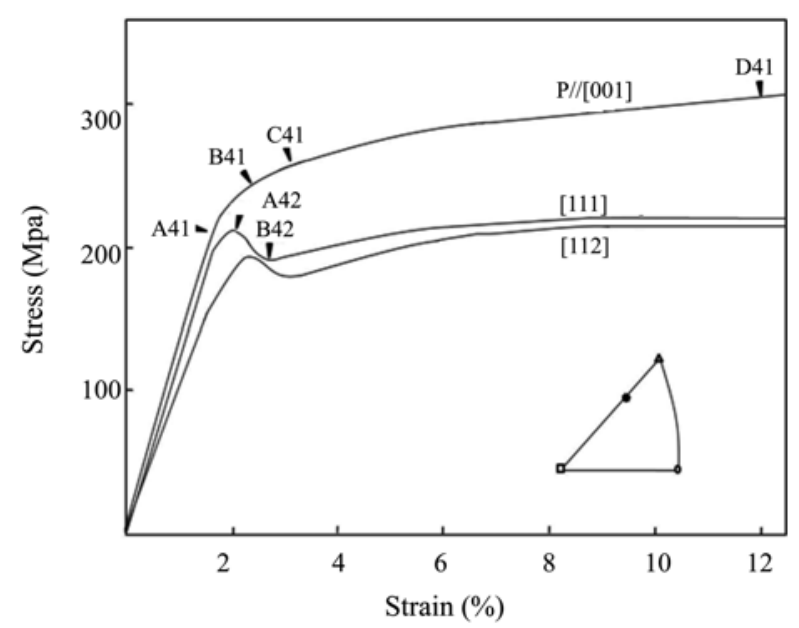

Fig. 1. Stress-Strain curves of the sample deformed along three different orientations, [112], [111] and [001] at $1400^{\circ} \mathrm{C}$. The three orientations are shown in the standard stereographic triangle. The arrowed stage $A_{41}$ to $D_{41}$ and $A_{42}$ and $B_{42}$ are where the deformation experiments were stopped and dislocation structures studied by TEM.

\section{Results and discussion}

$\mathrm{ZrO}_{2}$ exhibits plastic deformation by the activation of dislocation movements at high temperatures. Y-CSZ has cubic fluorite structure, and the space group is $F_{m 3 m}$. Anions occupy all eight tetrehedral sites and cations form a FCC sublattice. The plastic behavior of this crystal is highly dependent on crystallographic orientation. When the crystals were deformed along different orientations, plastic behaviors were changed by showing different stress-strain curves. Fig. 1 shows the stress-strain curves for the samples deformed along three different orientations, $<112>,<111>$ and $<001>$. Three different orientations of compression sample are also displayed in the standard stereographic triangle. When the samples were deformed along $<112>$, yield drop occurred which was caused by the activation of secondary slip system.[1, 2] The samples oriented to $<111>$ activated three $\{001\}<110>$ slip systems, as shown in Fig. 2, which showed the slip plane (marked by P) and Burgers vector (marked by $b$ ). These three slip systems have the same Schmid factor of 0.45 . The characteristic of stress-strain curve of this sample was very similar to that of the sample deformed along $<112>$ by showing yield drop and zero-work hardening regime. But the strength of the sample deformed along $<111>$ was slightly higher than that of the sample deformed along $<112>$.

However the nature of the stress-strain curve was changed when the samples were deformed along $<001>$. With this orientation, eight $\{111\}<110>$ slip system were

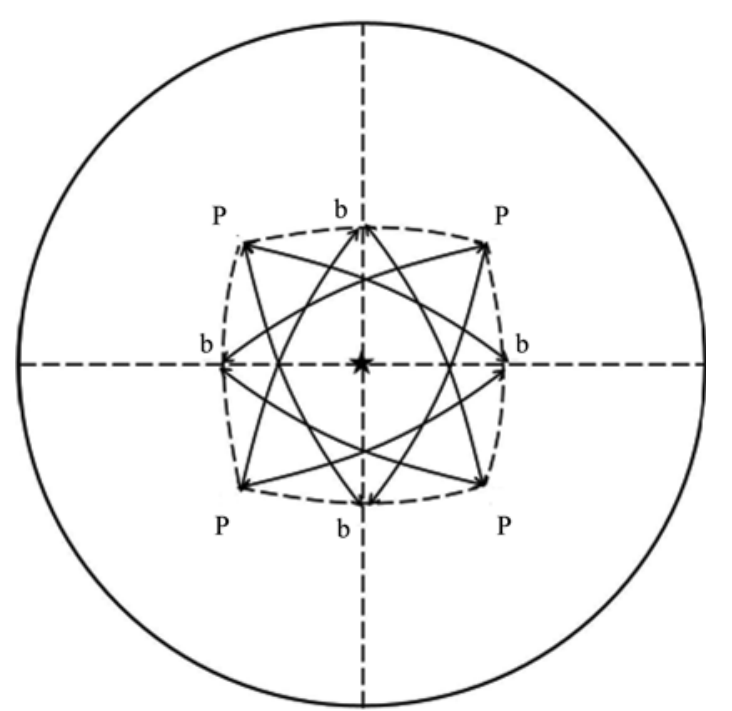

Fig. 2. Stereographic projection of the three $\{001\}<110>$ slip systems which are equally activated on [111] projection, Slip plain and Burgers vectors marked by $\mathrm{p}$ an $\mathrm{b}$, respectively. 


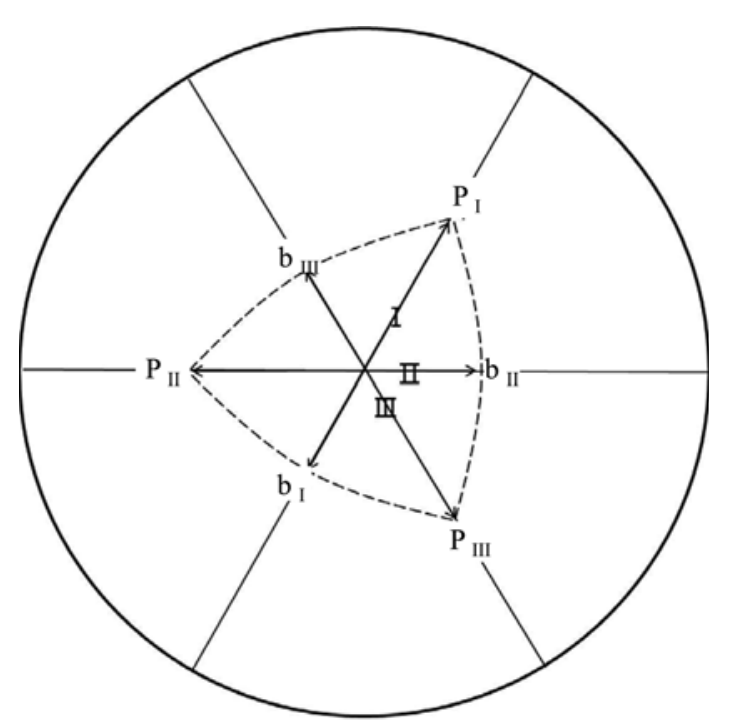

Fig. 3. Stereographic projection of the multiple $\{111\}<110>$ slip systems on [001] projection. Eight slip systems have the same Schmid factor. Slip plain and Burgers vectors marked by $\mathrm{p}$ an $\mathrm{b}$, respectively.

activated, as demonstrated in the stereographic projection in Fig. 3. The Schmid factor of all these slip systems have the same value of 0.45 . And yield drop was disappeared in this orientation. And the strength of this sample was higher than those of two other orientations. In other word,

$$
\sigma_{001}>\sigma_{111}>\sigma_{112}
$$

When the samples deformed along $<112>$ and $<111>$, both of which showed yield drop and activated $\{001\}$ $<110>$ slip systems. This results is consistent with the deformation results of $\left.\mathrm{UO}_{2}{ }^{9}{ }^{9}\right)$ Fries et al. ${ }^{1)}$ reported the consistent results with this crystal that the sample deformed along $<112>$ and $<111>$ exhibit yield drop but not yield drop for the sample deformed along $<011>$. However their yield stresses for the sample deformed along $<112>$ and $<111>$ were higher than one deformed along $<001>$.

The samples which were deformed to yield point along $<111>$ showed very clear slips on $\{110\}$ and $\{112\}$ planes, as shown in Fig. 4. $\theta$ and $\phi$ are the angles of the slip plane inclination with respect to the compression axes on $\{110\}$ and $\{112\}$ side surfaces, respectively. Measured value of $\theta$ and $\phi$ are 60 and $45^{\circ}$, respectively, which are agreed well with the calculated value. But slip trace results showed that only one $\{001\}<110>$ slip system is operating. It is not clear that only one slip system was activated instead of three slip systems. Even though the reason for the inhomogeneous deformation is not clear, it may be caused by the feasible experimental error of orienting

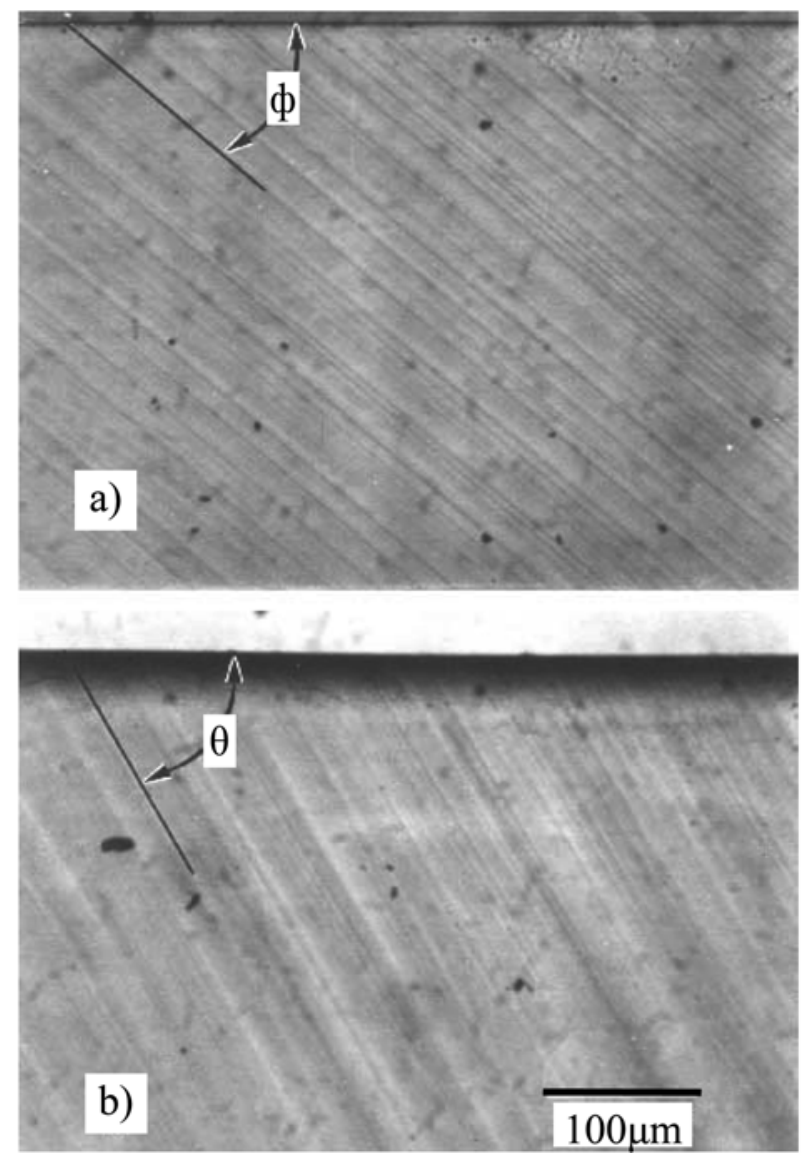

Fig. 4. Slip traces of the sample deformed along $<111>$. The slip lines on $\{110\}$ and $\{112\}$ side surfaces have (b) $65^{\circ}$ and (a) $40^{\circ}$ with respect to the compression axes, respectively.

compression axes, less than few degrees off from $<111>$.

The sample deformed along $<001>$ near yield point was etched and exhibited traces of dislocation etch pits as shown in Fig. 5. All traces of etch pits on both surfaces, $\mathrm{r}$ and $\theta$ in the $\{010\}$ plane, and $\Omega$ and $\phi$ in the $\{100\}$ plane are inclined $45^{\circ}$ with respect to the loading axes, $\mathrm{P} / /<001>$, which agreed with theoretical value. This results indicate that all four possible slip planes are operating under yield stress.

Dislocation substructures of the sample deformed along $<111>$ at upper yield point were similar to those of the sample deformed along $<112>$. The dislocation structure at this stage was composed of many edge-type dipoles and loops, as shown in Fig. 6. Node formation (Marked as $\mathrm{N})$ were also observed, indicating the activation of other slip systems, following the reaction,

$$
1 / 2[001]+1 / 2[10-1] \rightarrow 1 / 2[110]
$$

The nodes were frequently observed in the samples deformed along the other orientations. At the lower yield 

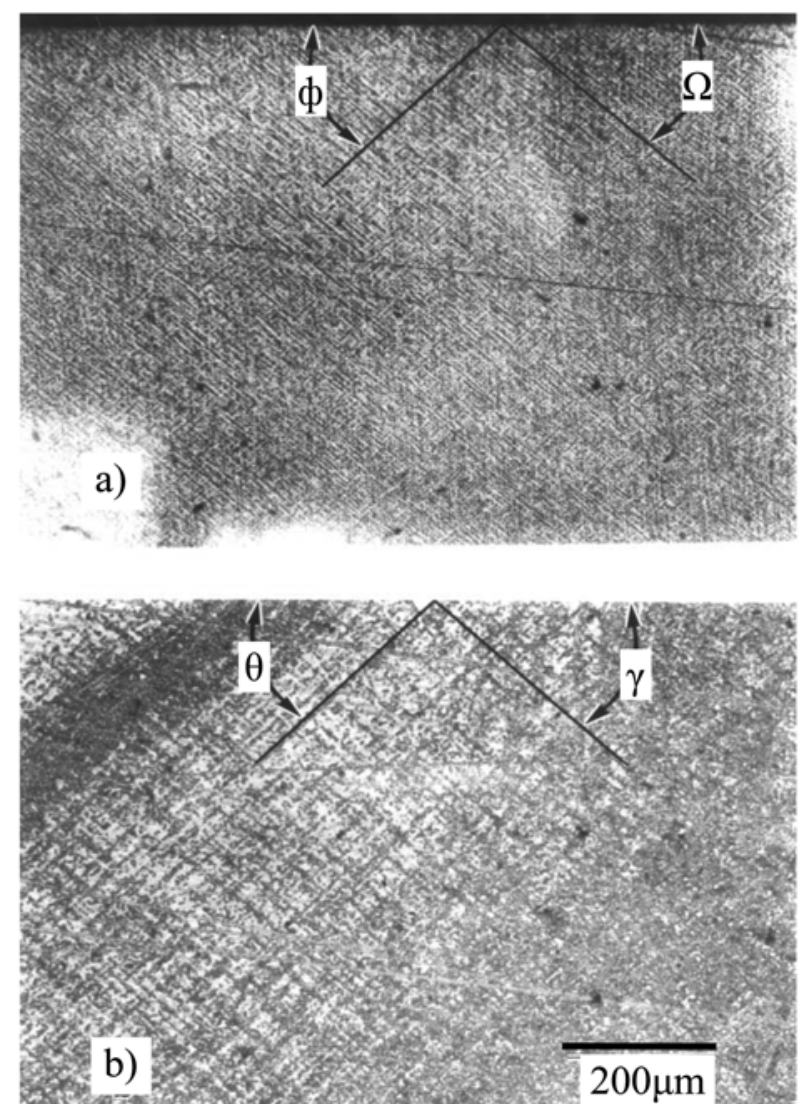

Fig. 5. Dislocation etch pits and slip lines on two different surfaces of the sample deformed along [001]. Four slip lines make $45^{\circ}$ (Marked $\mathrm{r}, \theta, \Omega, \phi$ ) with respect to the compression axes.

point, more secondary dislocations and nodes were observed while the nature of primary dislocations was very similar to that of the sample deformed to the lower yield point, as shown in Fig. 7. When samples were deformed along $<111>$ to about the yield point, the sample exhibited low density of dislocation compared to the samples deformed along $<112>$ and $<001>$, which showed yield drops. However all six Burgers vectors were observed in the very early stage of plastic deformation in the sample deformed along $<111>$ as shown in Fig. 8. At this stage, nodes were also formed by the same type of dislocation reaction following the equation. The surprising result at this stage was the dislocation interaction; two unit dislocations combined and formed a dislocation with the Burgers vector of [100], following the equation.

$$
1 / 2[110]+1 / 2[1-10] \rightarrow[100]
$$

The dislocation interaction does not result in any change of dislocation line energy. Since the Burgers vector of the resulting dislocation is very large, equal to the lattice

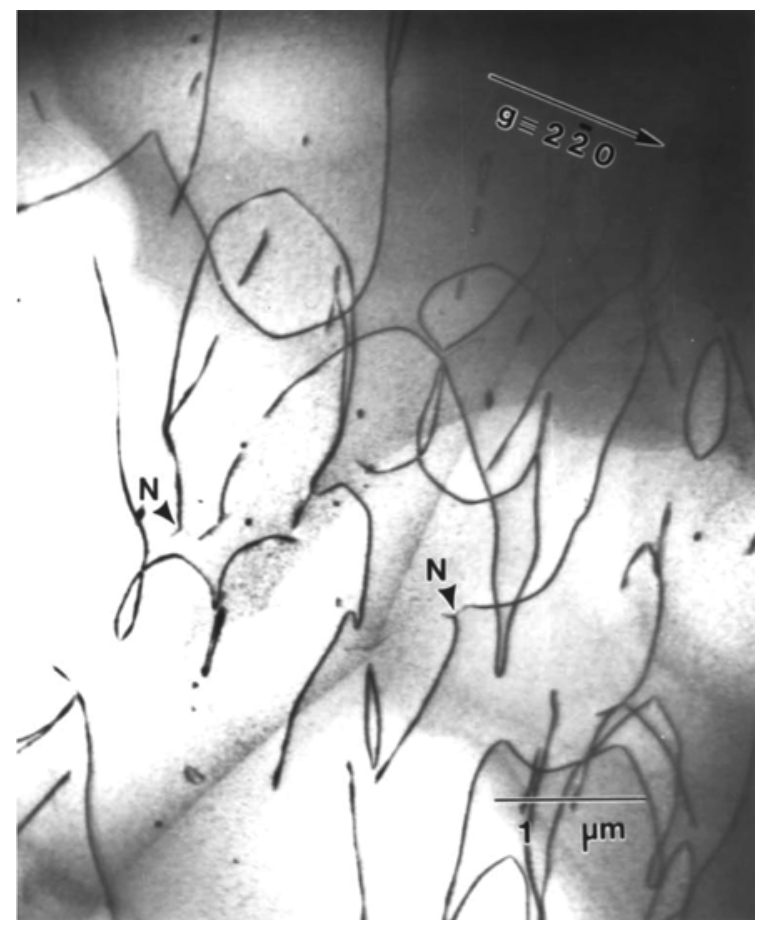

Fig. 6. Dislocation structures on (001) plane of the sample deformed to the stage $\mathrm{A}_{42}$. Most dislocations were edge-type nodes, marked by $\mathrm{N}$.

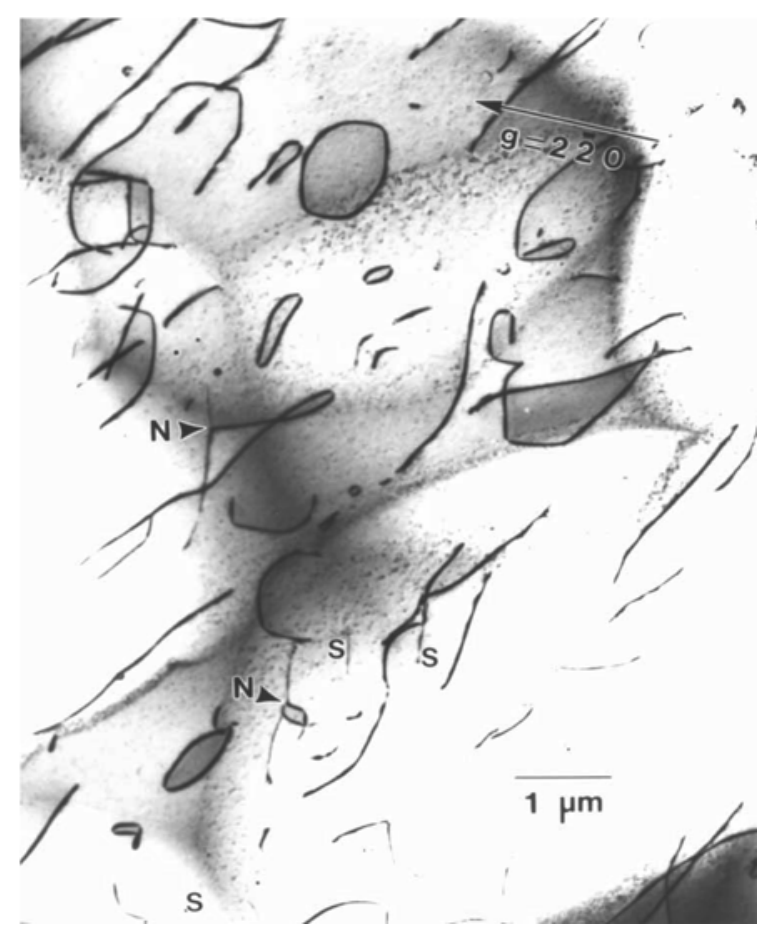

Fig. 7. Dislocation structures of the sample deformed to the stage $\mathrm{B}_{42}$. There are some nodes and secondary dislocations observed on (001) plane.

parameter, this dislocation must be very sessile. Since the sessile dislocations also do not exist on the glide plane 


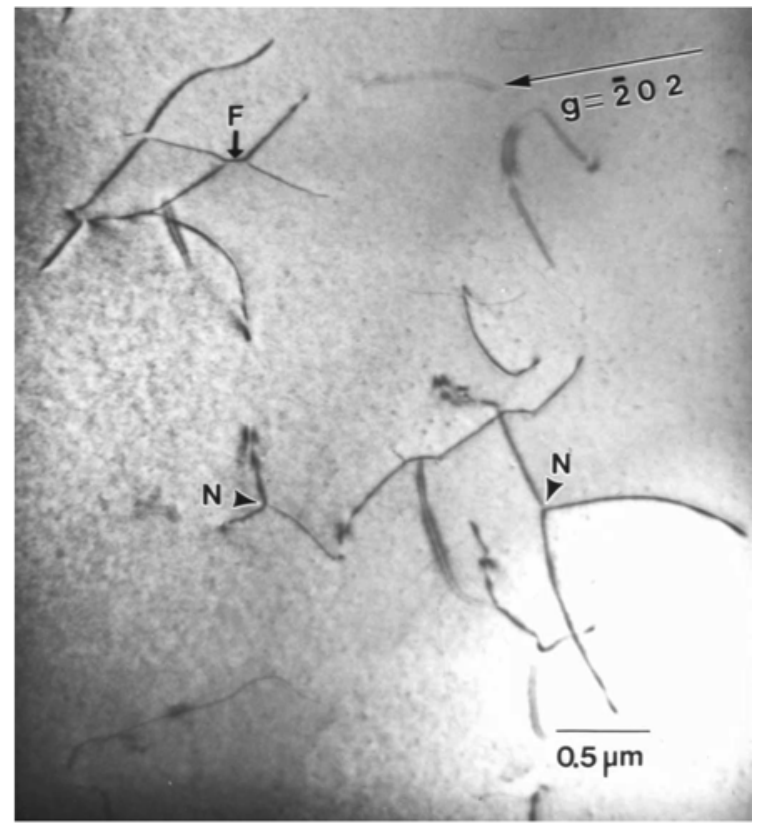

Fig. 8. Dislocation structures of the sample deformed to the stage $\mathrm{A}_{41}$. All six $<110>$ type Burgers vectors were observed. And nodes, $\mathrm{N}$ and strong sessile dislocations, $\mathrm{F}$ were observed on $\{111\}$ plane.

and the Schmid factor is zero, these sessile dislocations interrupt the movement of other dislocations and increase the work hardening rate, as shown in Fig. 1. This Diffraction contrast experiments of the sessile dislocations were explained by Cheong. ${ }^{10)}$ Philips ${ }^{11)}$ reported the same type of dislocation interaction in $\mathrm{CaF}_{2}$ and suggested that the $<100>$ type dislocation nucleated the fracture of the crystal. The $<100>$ type dislocations were also observed in $\mathrm{UO}_{2}$ which was deformed along [029]. ${ }^{12)}$ This sessile dislocation formed by the reaction of dislocations on two $\{111\}<110>$ slip systems in $\mathrm{UO}_{2}$ crystal.

Another $\{111\}$ foil was prepared from the same sample to study the three dimensional dislocation structures. The dislocation density was negligible on the second $\{111\}$ foil, implying that the dislocations were not formed uniformly during the early stage of plastic deformation in the sample deformed along $<001>$. Slip trace analysis showed all four slip planes which have the same Schmid factors, as shown in Fig. 5. However, these eight slip systems were operating during the later stage of plastic deformation of the sample. The sample deformed up to the $10 \%$ of plastic deformation showed no appreciable change of dislocation structures, but slight increase of dislocation density implying the slight work harding in stress-strain curve in Fig. 1. At this stage, six $<110>$ type Burgers vectors were observed

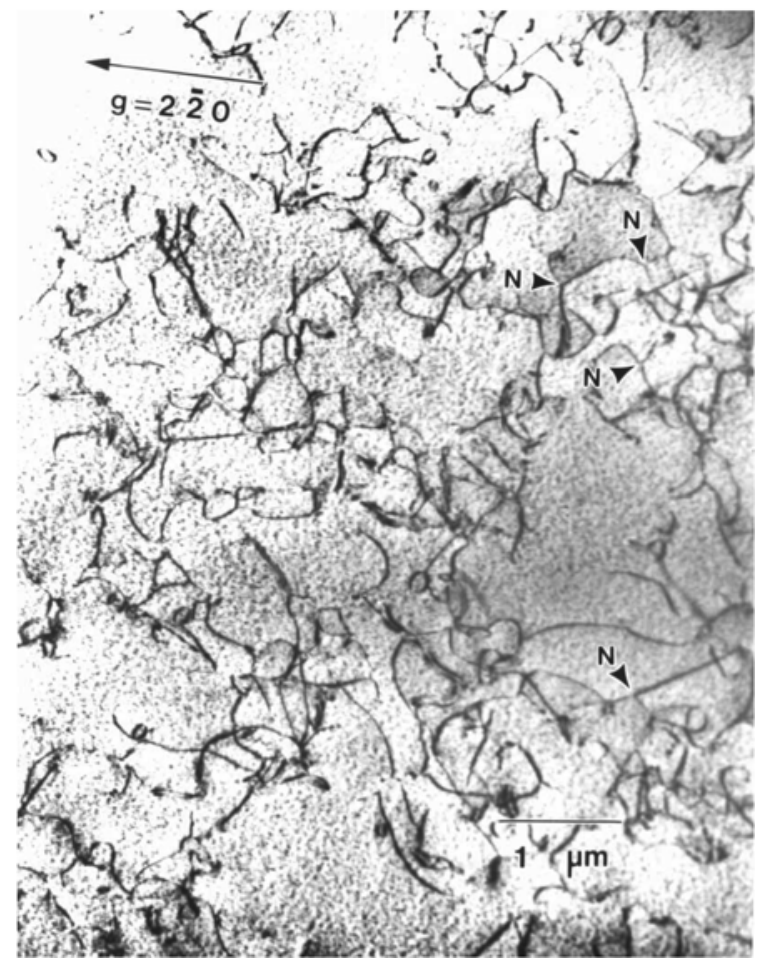

Fig. 9. Dislocation structures of the sample deformed to the stage $\mathrm{D}_{41}$. Many dislocation nodes, tangles and networks were formed by dislocation interaction in various slip systems.

with random orientations. The distribution of dislocations became more homogeneous as deformation proceeded. From the sample deformed to the stage $\mathrm{D}_{41}$, TEM foils parallel to three different $\{111\}$ planes showed the similar dislocation structures by showing dislocation networks, tangles and nodes, as shown in Fig. 9. It may indicate uniform plastic deformation at later stage of deformation in this sample.

Thus through comparisons of yield drop and zero work hardening regions, it may clear that the plastic behavior of Y-CSZ single crystals deformed along $<111>$ is very similar to that of the sample deformed along $<112>$. However the crystal deformed along $<001>$ displayed higher work hardening and no yield drop. Plastic deformation was carried out primarily on the only one slip plane, while all six $<110>$ type Burgers vectors were operating during the beginning of the plastic deformation. Due to the interaction of the two $1 / 2<110>$ dislocations, a strong $<100>$ sessile dislocations were formed. This sessile dislocations may cause the absence of yield drop in the early stage and higher work hardening in the later stage of plastic deformation. In the later stages of plastic deformation, all four slip planes were operated uniformly. 
As plastic deformation progressed, density of dislocations were increased and the dislocations with many slip systems were operating through convention dislocation multiplication. $^{13)}$ At this stage this sample showed dislocation networks with many dislocation nods which caused workhardening at the stage $\mathrm{D}_{41}$. The dislocation network and nods indicate that multiple slip system was activated, as shown in Fig. 9. As plastic deformation progressed to the stage, more than $10 \%$ of strain, deformation became uniform with dislocation reaction by activating more slip systems.

\section{Conclusion}

Y-CSZ single crystal showed plastic deformation which was dependent on crystallographic orientation. The slip systems in the sample deformed along $<112>,<111>$ and $<001>$ followed the theoretical values of the plastic deformation. Samples oriented to activate $\{001\}<110>$ slip system displayed a yield drops, while the sample deformed along $<001>$ to activate $\{111\}<110>$ did not showed yield drop and higher work hardening. It might be caused by the strong sessile dislocation. The strength of samples without yield drop showed higher strength than those of sample exhibiting yield drop.

At the early stage of plastic deformation, all sample exhibited dislocation dipoles and in the later stage, dislocation interactions occurred by forming nodes, tangles and networks. As plastic deformation progressed, more slip systems were activated and uniform dislocation structures were observed.

\section{References}

1. E. Fries, F. Guiberteau, A. Dominguez-Rodriguez, D. -S. Cheong, and A. H. Heuer, Phil. Mag. A, 60(1), 107 (1989).

2. A. Dominguez-Rodriguez, D. -S. Cheong, and A. H. Heuer, Phil. Mag. A, 64(4), 923 (1991).

3. D. -S. Cheong, A. Dominguez-Rodriguez, and A. H. Heuer, Phil. Mag. A, 60(1), 123 (1989).

4. W. G. Johnston, J. Appl. Phys., 33(9), 2050 (1962).

5. P. B. Hirsch and T. E. Mitchell, Work Hardening of Metals, edited by J. P. Hirth and J. Weertman, Gordon Beach, Science Publishers, Inc. N. Y. (1968).

6. D. -S. Cheong, A. Dominguez-Rodriguez, and A. H. Heuer, Phil. Mag. A, 63(3), 377 (1991).

7. D. -S. Cheong, C. -S. Kim, Submitted to J. Kor. Cry. Grow. and Crys. Tech. (2009).

8. P. B. Hirsch, A. Howie, R. B. Nicholson, D. W. Pashley and M. J. Whelan, Electron Microscopy of Thin Crystal, Robert E. Krieger Pub. Co., Huntington, N. Y. (1977).

9. P. T. Sawbridge and E. C. Sykes, Phil. Mag. 22(1), 33 (1971).

10. D. -S. Cheong, Ph. D Thesis, Case Western Reserve University. (1989).

11. W. L. Philips, J. Am. Ceram. Soc. 44(10) 499 (1961).

12. R. J. Keller, T. E. Mitchell and A. H. Heuer, Acta. Metall. 36(4), 1061 (1988).

13. J. J. Gilman and W. G. Johnston, Solid State Phys., 13(2), 245 (1963). 